МЕЛАТОНИНОВЫЙ СТАТУС У ПАЦИЕНТОК С ОЖИРЕНИЕМ И ДИСФУНКЦИЕЙ ЯИЧНИКОВ В РЕПРОДУКТИВНОМ ВОЗРАСТЕ

(С Е.Н. Андреева ${ }^{1,2}$, О.Р. Григорян ${ }^{1}$, Ю.С. Абсатарова ${ }^{1 *}$, Е.В. Шереметьева' ${ }^{1}$ Р.К. Михеев

'Национальный медицинский исследовательский центр эндокринологии, Москва, Россия

${ }^{2}$ Московский государственный медико-стоматологический университет им. А.И. Евдокимова, Москва, Россия

ОБОСНОВАНИЕ. Мелатонин - главный гормон эпифиза. Регулируя циркадианные ритмы, являясь иммунорегулятором и антиоксидантом, данный гормон принимает участие в работе яичников: его высокие концентрации блокируют апоптоз ооцитов и нейтрализуют активные формы кислорода, участвующие в фолликулогенезе, овуляции, созревании яйцеклетки и образовании желтого тела.

ЦЕЛЬ. Изучить мелатониновый статус и его взаимосвязь с менструальной дисфункцией и нарушениями сна у пациенток репродуктивного возраста с ожирением.

МАТЕРИАЛЫ И МЕТОДЫ. В одномоментном сравнительном исследовании приняли участие женщины 18-35 лет: 30 пациенток с ожирением и нарушениями менструальной функции неорганического характера и 30 здоровых женщин группы сравнения с нормальной массой тела и регулярным менструальным циклом. Все участницы прошли анкетирование для выявления сомнологических нарушений, а также был исследован уровень мелатонина в слюне и 6-сульфатоксимелатонина в моче.

PЕзУЛЬтАтЫ. В группе пациенток с ожирением $(n=30)$ в 47\% случаев встречались различные нарушения сна ( $p=0,003)$, в том числе чаще регистрировали синдром обструктивного апноэ сна (30\% случаев), а также была обнаружена корреляция между показателями анкетирования субъективных характеристик сна и индексом массы тела пациенток $(r=0,450 ; p=0,030)$ по сравнению с группой здоровых женщин с нормальной массой тела $(n=30)$. B основной группе уровень мелатонина в слюне оказался статистически значимо ниже по сравнению с контролем: медиана 12,6 и 25,5 пг/мл соответственно ( $p=0,008)$, та же закономерность была зарегистрирована для 6-сульфатоксимелатонина: 14,72 и 31,12 пг/мл соответственно.

ЗАКЛючЕНИЕ. Пациентки с ожирением и менструальной дисфункцией чаще страдают различными нарушениями сна и имеют более низкие показатели мелатонина в слюне и 6-сульфатоксимелатонина в моче.

КЛЮЧЕВЫЕ СЛОВА: мелатонин; ожирение; менструальная дисфункция; нарушения сна.

\title{
MELATONIN STATUS IN OBESE PATIENTS WITH OVARIAN DYSFUNCTION AT REPRODUCTIVE AGE
}

(c) Elena N. Andreeva ${ }^{1,2}$, Olga R. Grigoryan' ${ }^{1}$, Yuliia S. Absatarova ${ }^{1 *}$, Ekaterina V. Sheremetyeva', Robert K. Mikheev ${ }^{1}$

'Endocrinology Research Centre, Moscow, Russia

${ }^{2}$ A.I. Evdokimov Moscow State University of Medicine and Dentistry, Moscow, Russia

BACKGROUND: Melatonin is the main hormone of the pineal gland. By regulating circadian rhythms and being an immune regulator and antioxidant, this hormone takes part in the work of the ovaries: its high concentrations block apoptosis and neutralize reactive oxygen species involved in folliculogenesis, ovulation, egg maturation and corpus luteum formation. AIM: To study melatonin status and its relationship with menstrual dysfunction and sleep disorders in obese women of reproductive age.

MATERIALS AND METHODS. In a one-stage comparative study, women 18-35 years old took part: 30 patients with obesity and menstrual disorders of an inorganic nature and 30 healthy women in the comparison group with normal weight and regular menstrual cycle. All participants underwent a questionnaire to identify somnological disorders, and the level of melatonin in saliva and 6-sulfatoxymelatonin in urine was also investigated.

RESULTS: In the group of patients with obesity $(n=30)$, various sleep disorders were encountered in $47 \%$ of cases $(p=0.003)$, including more often obstructive sleep apnea syndrome was recorded ( $30 \%$ of cases), and a correlation was found between the indicators of the questionnaire survey of subjective sleep characteristics and body mass index of patients ( $r=0.450$, $p=0.030)$ compared with a group of healthy women with normal weight $(n=30)$. In the main group, the level of melatonin in saliva was statistically significantly lower than in the control: median $12.6 \mathrm{pg} / \mathrm{ml}$ and $25.5 \mathrm{pg} / \mathrm{ml}$, respectively ( $\mathrm{p}=0.008$ ), the same pattern was recorded for 6-sulfatoxymelatonin: $14,72 \mathrm{pg} / \mathrm{ml}$ and $31.12 \mathrm{pg} / \mathrm{ml}$, respectively.

CONCLUSION: Patients with obesity and menstrual dysfunction are more likely to suffer from various sleep disorders and have lower levels of melatonin in saliva and 6-sulfatoxymelatonin in urine.

KEYWORDS: melatonin; obesity; menstrual dysfunction; sleep disorders. 


\section{ОБОСНОВАНИЕ}

Мелатонин - гормон, который в основном синтезируется в ткани эпифиза в ночное время и контролирует циркадианные ритмы млекопитающих [1]. Он также вырабатывается в других тканях и органах: кишечнике, яичниках, сетчатке и других [2]. Помимо регулирующей циркадианные ритмы функции, мелатонин обладает антиоксидантными, противовоспалительными, антиапоптотическими и регенерирующими свойствами. Исследования показывают, что данный гормон влияет на функцию жировой ткани, метаболизм липидов и массу тела [3].

Ожирение - одна из самых распространенных медицинских проблем в развитых странах во всех возрастных группах, среди и мужчин, и женщин [4]. Рост распространенности избыточной массы тела и ожирения - серьезная угроза общественному здоровью во всем мире. В последнее время внимание исследователей привлекает возможная роль мелатонина в регуляции массы тела и предотвращении метаболических нарушений, связанных с ожирением.

Механизм регулирующего влияния мелатонина на массу тела довольно сложен. Во-первых, гормон контролирует дифференцировку адипоцитов и адипогенез [5]. Во-вторых, мелатонин индуцирует образование «бежевых» адипоцитов и потемнение белой жировой ткани, увеличивая термогенную способность и расход энергии, препятствуя тем самым увеличению массы тела [6]. В-третьих, мелатонин способствует повышенному образованию бурой жировой ткани и активации ее функции [7]. Еще один возможный механизм связывают с изменением микробиоты кишечника: на экспериментальных моделях на фоне дотации экзогенного мелатонина было продемонстрировано преобладание микробных сообществ, ассоциированных с позитивными изменениями липидного и энергетического метаболизма [8]. Следует учитывать также антиоксидантные и противовоспалительные свойства гормона, который потенцирует снижение массы тела, устраняя дисфункцию адипоцитов и митохондрий [9]. Дополнительный прием мелатонина восстанавливал секрецию и метаболизм адипонектина у мышей с ожирением, что тоже может объяснить его защитный эффект в отношении увеличения массы тела [4].

Регуляторный механизм действия мелатонина варьирует в зависимости от органа-мишени. В поджелудочной железе гормон снижает уровни циклического аденозинмонофосфата (цАМФ) и циклического гуанозинмонофосфата (цГМФ), через которые осуществляется контроль за гомеостазом инсулина и глюкозы [10]. В результате этого процесса происходят ритмические колебания чувствительности тканей к глюкозе, максимальной в утренние часы и минимальной ночью [11]. Что касается других физиологических эффектов, то антиоксидантная способность мелатонина в основном связана с увеличением производства антиоксидантных ферментов, включая супероксиддисмутазу, каталазу, глутатион, а также с его антиоксидантным действием как таковым [12].

Известно, что депривация сна - один из факторов риска развития ожирения [4]. Эпидемиологические исследования выявили взаимосвязь между хроническим недосыпанием и увеличением массы тела [13]. Цирка- дианные ритмы являются фундаментальными биологическими процессами, которые регулирует цикл «деньночь», обеспечивая периодичность биохимических, молекулярных и физиологических процессов. Появляется все больше доказательств связи системы биологических часов, метаболизма и энергетического баланса [13]. Изменения в образе жизни, такие как увеличение времени бодрствования, использование искусственного света, телевидения, компьютерных мониторов и других средств массовой информации, нарушают циркадианные ритмы. Одним из последствий является нарушение режима секреции мелатонина. В клинических исследованиях была обнаружена обратная связь между уровнем мелатонина и степенью ожирения [4]. Снижение уровня гормона усугубляет инсулинорезистентность и выраженность метаболического синдрома [14].

Регулируя циркадианные ритмы и являясь антиоксидантом, мелатонин, в том числе, принимает участие в работе яичников: его высокие концентрации блокируют апоптоз ооцитов и нейтрализуют активные формы кислорода, участвующие в фолликулогенезе, овуляции, созревании яйцеклетки и образовании желтого тела. Данный гормон является одним из ключевых нейроэндокринных регуляторов работы оси гипоталамус-эпифиз-гонады, влияя на синтез и секрецию гонадотропинов [15]. Было показано, что мелатонин увеличивает экспрессию белков кисспептинов, которые являются важными регуляторными молекулами в секреции лютеинизирующего и фолликулостимулирующего гормонов $[16,17]$. Положительный эффект «гормона сна» на фертильность, развитие ооцитов и поддержание эмбриона объясняется его способностью уменьшать окислительное повреждение фолликулов яичников. В то же время более высокие концентрации мелатонина, наблюдаемые во время беременности, по-видимому, поддерживают созревание плода и гомеостаз плаценты [18].

Одним из частых коморбидных состояний у пациенток с ожирением является менструальная дисфункция. Сопутствующий дефицит мелатонина у женщин с повышенным индексом массы тела (ИМТ) может непосредственно усугублять нарушения менструального цикла. Антиоксидантные свойства мелатонина и его влияние на ось гипоталамус-эпифиз-яичники способствуют уменьшению окислительного повреждения фолликулов, улучшая продукцию прогестерона в лютеиновой фазе цикла и созревание ооцитов [19]. Циркулирующий мелатонин может поглощаться яичником, при этом фолликул сам обладает способностью синтезировать и секретировать собственный гормон, тем самым демонстрируя его важную паракринную роль в женской репродуктивной системе [20]. На экспериментальных моделях было продемонстрировано, что после пинеалэктомии развивается овуляторная дисфункция, а добавление экзогенного мелатонина в питание в течение 12 мес улучшает количество и качество ооцитов, а также увеличивает размер помета [21, 22].

В клиническом исследовании на группе пациенток с синдромом поликистозных яичников, который часто сопровождается метаболическими и репродуктивными нарушениями, был продемонстрирован положительный эффект дотации экзогенного мелатонина на менструальную функцию и андрогенный профиль [23]. 
Антиандрогенный потенциал нейрогормона подтверждает его способность снижать уровень цАМФ в гранулезных клетках, что в конечном итоге приводит к уменьшению выработки мужских гормонов [15].

Косвенно оценить уровень мелатонина в крови позволяет измерение его уровня в слюне и его метаболита 6-сульфатоксимелатонина в моче, при этом ИМТ может оказывать значимое влияние на вышеуказанные параметры [24].

\section{ЦЕЛЬ ИССЛЕДОВАНИЯ}

Изучить мелатониновый статус и его взаимосвязь с менструальной дисфункцией и нарушениями сна у пациенток репродуктивного возраста с ожирением.

\section{МАТЕРИАЛЫ И МЕТОДЫ}

Место проведения. Исследование было выполнено на базе ФГБУ «НМИЦ эндокринологии» Минздрава России. Время исследования. Июль 2020 г. - апрель 2021 г.

Изучаемые популяции: 2 популяции (пациентки с ожирением и группа контроля - здоровые женщины).

\section{Популяция «пациентки с ожирением»}

Критерии включения: женщины 18-35 лет; ИМТ $\geq 30$ кг/м²; нарушения менструального цикла неорганического характера; отсутствие любой гормональной терапии или препаратами мелатонина в течение 6 мес перед исследованием; получение информированного согласия на комплексное обследование.

Критерии исключения: беременность, лактация; наличие хронических экстрагенитальных заболеваний в стадии обострения, субкомпенсации; наличие декомпенсированной эндокринной патологии; выявление острых и обострений хронических воспалительных заболеваний половых органов; курение, злоупотребление алкоголем.

\section{Популяция «здоровые женщины»}

Критерии включения:ИМТ 19,5-25,0 кг/м², наличие регулярного менструального цикла; отсутствие какой-либо гормональной терапии или препаратами мелатонина в течение 6 мес до исследования, получение информированного согласия на комплексное обследование.

Критерии исключения: наличие хронических экстрагенитальных заболеваний в стадии обострения, субкомпенсации; наличие декомпенсированной эндокринной патологии; выявление острых и обострений хронических воспалительных заболеваний половых органов; курение, злоупотребление алкоголем.

Выборка была сформирована путем сплошного включения наблюдений.

Дизайн исследования: одноцентровое одномоментное сравнительное исследование.

Описание медицинского вмешательства. Проведение клинико-лабораторного обследования: анкетирование (анкета балльной оценки субъективных характеристик сна, анкета скрининга синдрома апноэ во сне, Эпвортская шкала оценки дневной сонливости), гормональное исследование (определение уровня мелатонина в слюне в 3 ч ночи, определение уровня метаболита мелатонина 6-сульфатоксимелатонина в суточной моче).

\section{Методы}

В основную группу входили пациентки с различными нарушениями менструального цикла (частые менее 21 дня, редкие менструации - более 35 дней, аменорея) и ИМТ $\geq 30$ кг/M². В группу сравнения были включены здоровые женщины с регулярным менструальным циклом продолжительностью от 21 до 35 дней и ИМТ в пределах 19,5-25 кг/м². Все участницы прошли анкетирование с помощью анкеты балльной оценки субъективных характеристик сна (оценка продолжительности сна, времени засыпания, количества сновидений и ночных пробуждений, качества утреннего пробуждения). Показатель более 22 баллов говорил об отсутствии нарушений, пограничные значения колебались от 19 до 21 баллов, значение менее 19 баллов расценивали как расстройства сна [25]. Анкета скрининга синдрома апноэ во сне использовалась для оценки риска данного состояния: при показателе более 4 баллов диагностировали синдром обструктивного апноэ. Для оценки дневной сонливости применяли Эпвортскую шкалу (в норме показатель менее или равен 9 баллам).

Все участницы осуществляли самостоятельный сбор слюны дома в специальные контейнеры со стерильным тампоном в 3 ч ночи при отсутствии или минимальном освещении (слабый ночник). Тампон необходимо было поместить в ротовую полость, где он должен был находиться в течение 3 мин, далее без помощи рук его надо было поместить обратно в пробирку. Емкость можно было хранить в обычном холодильнике при температуре не выше $+6^{\circ} \mathrm{C}$. Контейнер доставляли на следующий день для исследования методом ELISA.

Сбор суточной мочи для определения 6-сульфатоксимелатонина методом ELISA осуществлялся в течение дня, начиная со второй порции после ночного сна в течение следующих суток. Емкость для сбора мочи хранилась в обычном холодильнике при температуре не выше $+6^{\circ} \mathrm{C}$. Для исследования была использована порция мочи 20 мл, в том числе учитывался общий суточный объем.

\section{Статистический анализ}

Статистическая обработка полученных результатов проводилась с помощью прикладных программ STATISTICA (StatSoft Inc. США, версия 6.0). Объем исследуемых групп был выбран с учетом проводимых ранее исследований аналогичного дизайна, а также положения о минимальном числе участников - 30 человек в одной исследуемой подгруппе. В связи с наличием небольших объемов выборки и распределения, отличающегося от нормального, использовались непараметрические методы статистики. U-критерий Манна-Уитни применяли для сравнения независимых групп по количественным признакам. Анализ таблиц сопряженности с использованием двустороннего точного критерия Фишера, а также критерия $X^{2}$ для несвязанных групп использовали для сравнения независимых групп по качественным признакам. Непараметрический метод ранговой корреляции по Спирмену применяли для анализа корреляции двух количественных признаков, а количественного и порядкового - по Кендаллу. Статистически значимыми считали различия при $\mathrm{p}<0,05$. 


\section{Этическая экспертиза}

Протокол исследования был одобрен этическим комитетом ФГБУ «НМИЦ эндокринологии» Минздрава России (№11 от 22.07.2020).

\section{РЕЗУЛЬТАТЬ}

Всего в исследовании приняли участие 60 женщин: 30 пациенток основной группы и 30 женщин группы сравнения (здоровые участницы). Пациентки были сопоставимы по возрасту и статистически значимо отличались по ИМТ (табл. 1).

При анализе данных анкет балльной оценки субъективных характеристик сна женщины с ожирением и нарушениями менструального цикла статистически значимо отличались от группы контроля по уровню патологических отклонений $(p=0,003)$ и чаще страдали сомнологическими нарушениями (табл. 2).

Мы обнаружили статистически значимую прямую корреляцию между количеством баллов согласно анкете балльной оценки субъективных характеристик сна и ИМТ в основной группе: чем выше был ИМТ, тем хуже были показатели сомнологического профиля по данным анкетирования $(r=0,450 ; p=0,030$, метод ранговой корреляции по Кендаллу).

По данным опросника, синдром обструктивного апноэ был статистически значимо более вероятен у пациенток с ожирением, чем в контрольной группе, хотя по уровню сонливости участницы были сопоставимы (табл. 3).

Анализ показателей мелатонина в слюне и его метаболита в суточной моче показал, что пациентки с ожирением и нарушениями менструального цикла имели более низкие значения изучаемых параметров по сравнению с группой здоровых женщин (табл. 4).

При корреляционном анализе была выявлена статистически значимая прямая корреляция уровня 6-сульфатоксимелатонина в суточной моче и мелатонина в слюне в 03.00 ночи $(r=0,850 ; p=0,020$, метод ранговой корреляции по Спирмену), а также уровня мелатонина в слюне и степени нарушения сна по данным анкеты балльной оценки субъективных характеристик сна ( $r=0,640 ; p=0,03$, метод ранговой корреляции по Спирмену).

\section{ОБСУЖДЕНИЕ}

Ожирение и его осложнения представляют собой серьезную проблему во всем мире. В последние десятилетия механизмы, лежащие в основе прогрессирования ожирения, активно изучаются. Предрасполагающими к развитию заболевания факторами являются поведенческие (высококалорийная диета, низкая физическая активность и недосыпание) и внутренние (дисрегуляция жировой ткани, хроническое воспаление, окислительный стресс) [26]. В научном сообществе активно

Таблица 1. Распределение участниц исследования по индексу массы тела и возрасту

\begin{tabular}{lccc}
\hline \multicolumn{1}{c}{ Показатель } & Основная группа, $\mathbf{n = 3 0}$ & Группа контроля, $\mathbf{n = 3 0}$ & p (U-тест) \\
\hline ИМТ, кг/м² & $33,8[30,6 ; 40,1]$ & $20,9[20,0 ; 22,8]$ & 0,001 \\
\hline Возраст, лет & $25[21 ; 34]$ & $26[22 ; 35]$ & 0,865 \\
\hline
\end{tabular}

Таблица 2. Частота отклонений сна в группе ожирения и группе контроля $\left(p=0,003\right.$, критерий $\left.x^{2}\right)$

\begin{tabular}{lcc}
\hline \multicolumn{1}{c}{ Параметры сна } & Основная группа, n=30 & Группа контроля, $\mathbf{n = 3 0}$ \\
\hline Сон нарушен, $\mathrm{n}(\%)$ & $14(46,7 \%)$ & $5(16,7 \%)$ \\
\hline $\begin{array}{l}\text { Нормальный сон+пограничный } \\
\text { результат, } \mathrm{n}(\%)\end{array}$ & $16(53,3 \%)$ & $25(83,3 \%)$ \\
\hline
\end{tabular}

Таблица 3. Сомнологические характеристики участниц исследования

\begin{tabular}{lccc}
\hline \multicolumn{1}{c}{ Показатель } & Основная группа, $\mathbf{n = 3 0}$ & Группа контроля, $\mathbf{n = 3 0}$ & р (критерий Фишера) \\
\hline Апноэ во сне, $\mathrm{n}(\%)$ & $9(30)$ & $1(3)$ & 0,006 \\
\hline Наличие сонливости, $\mathrm{n}(\%)$ & $12(40)$ & $10(33)$ & 0,081 \\
\hline
\end{tabular}

Таблица 4. Мелатониновый статус участниц исследования

\begin{tabular}{|c|c|c|c|}
\hline Показатель & $\begin{array}{c}\text { Основная группа, } \\
\text { n=30 } \\
\text { Медиана, 25-75\% } \\
\text { процентили }\end{array}$ & $\begin{array}{c}\text { Группа контроля, } \\
\text { n=30 } \\
\text { Медиана, 25-75\% } \\
\text { процентили }\end{array}$ & p (U-тест) \\
\hline $\begin{array}{l}\text { 6-сульфатоксимелатонин } \\
\text { в суточной моче, мкг/24 ч }\end{array}$ & $\begin{array}{c}14,72 \\
{[13,08 ; 17,35]}\end{array}$ & $\begin{array}{c}31,12 \\
{[20,43 ; 46,82]}\end{array}$ & 0,008 \\
\hline $\begin{array}{l}\text { Мелатонин в слюне в } 3 \text { ч } \\
\text { ночи, пг/мл }\end{array}$ & $\begin{array}{c}12,6 \\
{[5,99 ; 23,40]}\end{array}$ & $\begin{array}{c}25,5 \\
{[8,60 ; 32,56]}\end{array}$ & 0,003 \\
\hline
\end{tabular}


изучается возможная связь между дефицитом мелатонина и ожирением. В некоторых работах были продемонстрированы многообещающие результаты относительно потенциальной роли мелатонина в профилактике ожирения и его осложнений [4].

В нашем исследовании был проведен анализ показателей мелатонинового обмена (мелатонин в слюне и 6-сульфатоксимелатонин в суточной моче) в зависимости от наличия ожирения и хронобиологических нарушений у пациенток с менструальной дисфункцией, а также у здоровых женщин группы контроля. Был изучен сомнологический профиль участниц, а также взаимосвязь этих параметров с уровнем мелатонина в моче и слюне. По данным исследования, пациентки основной группы (ожирение) чаще страдали сомнологическими нарушениями, в том числе синдромом обструктивного апноэ. Нами была обнаружена корреляция между нарушениями сна и ИМТ у женщин с нарушениями менструальной функции. Предположительно, как следствие сомнологических расстройств участницы 1-й группы имели пониженное содержание мелатонина в слюне и 6-сульфатоксимелатонина в суточной моче. В том числе, была обнаружена корреляция содержания гормона в слюне и степени нарушений сна по данным анкеты балльной оценки субъективных характеристик сна, что тоже может подтверждать влияние депривации сна на выработку нейрогормона в эпифизе. Данные закономерности были зарегистрированы и в работах других авторов. Согласно результатам D. Corbalán-Tutau и соавт., у женщин с метаболическим синдромом наблюдалась сниженная амплитуда суточного ритма мелатонина. Было установлено, что циркадианный ритм гормона имел обратную корреляцию с уровнями лептина в плазме - одним из основных метаболических гормонов [27].

R. Reiter и соавт. была обнаружена связь между депривацией сна и нарушенной секрецией мелатонина [28]. Интересно, что короткая продолжительность сна коррелировала со снижением уровня лептина и повышенными значениями ИМТ в когортном исследовании, проведенном S. Taheri и соавт. [29]. В работе R. Markwald и соавт. 5 дней недостаточного сна в группе из 16 здоровых взрослых добровольцев привели к энергетическому дисбалансу, нарушенному циркадианному ритму мелатонина и повышению уровня лептина [13].

Короткую продолжительность сна, определяемую как сон 6 ч или меньше за 24-часовой период времени, имеют треть взрослого населения [30]. Нарушения сна чаще встречаются у женщин, чем у мужчин, и часто совпадают с колебаниями половых гормонов [31]. Связь нарушенных циркадианных ритмов и менструальной дисфункции была зарегистрирована в исследованиях с участием женщин, работающих посменно или в ночное время. Участницы, имевшие вышеуказанный график работы, имели различные отклонения в длительности, обильности менструаций или продолжительности менструального цикла [32]. В недавнем проспективном исследовании, куда были включены 287 недавно нанятых на работу медсестер с посменной работой, были изучены показатели качества сна в дополнение к количественной оценке сменного графика работы. Нарушение менструального цикла определялось как наличие цикла менее 21 дня или более 35 дней по крайней мере один раз в течение 6-месячного периода наблюдения. Бессонница была связана с 2-кратным увеличением вероятности нарушений менструального цикла, а нерегулярность цикла увеличивалась по мере нарастания тяжести бессонницы [33].

Относительно небольшое количество эпидемиологических исследований изучали нарушения сна и фертильность женщин. В работе с участием 22 пациенток, перенесших экстракорпоральное оплодотворение (ЭКО), измерялись продолжительность и качество сна в течение одного цикла ЭКО с использованием актиграфа и ежедневных дневников [34]. Исследователи обнаружили линейную связь между общим временем сна и количеством извлеченных ооцитов. В исследование I.D. Wang и соавт. были включены пациентки из национального регистра: 16718 женщин с нарушениями сна и 33436 женщин без (за исключением апноэ во сне), после чего была изучена связь между сомнологическими расстройствами и бесплодием (неспособность зачать ребенка в течение 1 года) [35]. У участниц с нарушением сна риск бесплодия был в 3,7 раза выше, чем у женщин группы сравнения; этот результат прослеживался на протяжении 11 лет наблюдения. Другое проспективное когортное исследование с участием 6873 специалистов по планированию беременности в Северной Америке показало, что плохое качество сна, которое оценили с помощью опросников, было связано с более длительным сроком до наступления беременности [36]. Также наблюдалась слабая обратная корреляция между короткой продолжительностью сна и количеством родов, а сменная работа не была связана с рождаемостью.

Суррогатным биомаркером метаболизма мелатонина является 6-сульфатоксимелатонин, метаболит гормона, экскретируемый с мочой. Он используется для оценки мелатонинового статуса среди пациенток с различными гинекологическими заболеваниями [37]. Снижение уровня 6-сульфатоксимелатонина в моче, связанное с более высокими уровнями эстрадиола и прогестерона, было обнаружено у женщин, работающих в ночную смену, по сравнению с женщинами, выходящими в дневное время [38]. При этом R. Luboshitzky и соавт. обнаружили повышенный уровень 6-сульфатоксимелатонина в моче в группе женщин с диагнозом синдром поликистозных яичников по сравнению с контролем, что авторы связывают с влиянием андрогенов при данной нозологии [39].

Клиническая значимость наших результатов является высокой, так как полученные данные позволяют планировать более крупные будущие исследования для поиска эффективных и патогенетически обоснованных методов коррекции метаболических нарушений и менструальной дисфункции у пациенток репродуктивного возраста. Корреляция мелатонина в слюне и его метаболита в суточной моче позволяет использовать метод определения гормона в слюне в качестве быстрого и информативного теста для уточнения мелатонинового статуса женщины.

Ограничениями нашего исследования могут быть условия проведения, так как набор участниц проводился только в федеральном научном центре, выявление нарушений сна только на основании анкетирования, без использования объективных методов диагностики (сомнография).

Нормальный циркадианный ритм имеет важное значение для энергетического гомеостаза организма. 
Появляется все больше работ, подтверждающих положительное влияние дотации экзогенного мелатонина на пациенток с ожирения. Тем не менее механизмы, лежащие в основе этого эффекта, еще до конца не изучены. Возможно, антиоксидантная и противовоспалительная функции этого гормона определяют его эффективность в коррекции метаболических нарушений. Несомненно, необходимы более крупные исследования, которые позволят осветить весь механизм действия мелатонина у пациенток с ожирением и, возможно, использовать его как терапевтическое средство для профилактики избыточного веса и его осложнений, а также установить роль сомнологических нарушений в развитии неблагоприятных репродуктивных исходов.

\section{ЗАКЛЮЧЕНИЕ}

Качество и продолжительность сна могут иметь важное значение для репродуктивной функции женщины. Механизмы, лежащие в основе этой связи, вероятно, сложные и многофакторные. Нарушения циркадианного ритма могут влиять на овариальную функцию из-за сбоев в регуляции оси гипоталамус-эпифиз-яичники, в частности, за счет дефицита мелатонина и, как следствие, развившихся метаболических нарушений (резистентность к инсулину, окислительный стресс и системное воспаление). Мелатонин является потенциальным кандидатом для лечения ожирения за счет своего влияния на метаболизм инсулина и жировой ткани, липолиз и митохондриальную функцию. Гормон снижает дисфункцию жировой ткани благодаря своим антиоксидантным и противовоспалительным эффектам. Доказательства его защитных свойств и отсутствие значимых побочных реакций могут способствовать его продвижению в качестве терапевтического средства для пациентов с ожирением.

\section{ДОПОЛНИТЕЛЬНАЯ ИНФОРМАЦИЯ}

Источник финансирования. Работа выполнена в рамках Государственного задания «Центральные и периферические патофизиологические механизмы развития болезней жировой ткани с учетом клинических и гормональных характеристик», 2020-2022 годы.

Конфликт интересов. Авторы декларируют отсутствие явных и потенциальных конфликтов интересов, связанных с публикацией настоящей статьи.

Участие авторов. Все авторы одобрили финальную версию статьи перед публикацией, выразили согласие нести ответственность за все аспекты работы, подразумевающую надлежащее изучение и решение вопросов, связанных с точностью или добросовестностью любой части работы

\section{СПИСОК ЛИТЕРАТУРЫ | REFERENCES}

1. $\mathrm{Xu} \mathrm{Z,} \mathrm{You} \mathrm{W,} \mathrm{Liu} \mathrm{J,} \mathrm{et} \mathrm{al.} \mathrm{Elucidating} \mathrm{the} \mathrm{Regulatory} \mathrm{Role} \mathrm{of} \mathrm{Melatonin}$ in Brown, White, and Beige Adipocytes. Adv Nutr. 2019;46(5):847-852. doi: https://doi.org/10.1093/advances/nmz070

2. Luchetti F, Canonico B, Bartolini D, et al. Melatonin regulates mesenchymal stem cell differentiation: a review. J Pineal Res. 2014;56(4):382-97. doi: https://doi.org/10.1111/jpi.12133

3. Pandi-Perumal SR, BaHammam AS, Ojike NI, et al. Melatonin and Human Cardiovascular Disease. J Cardiovasc Pharmacol Ther. 2017;22(2):122-132. doi: https://doi.org/10.1177/1074248416660622

4. Szewczyk-Golec K, Woźniak A, Reiter RJ. Inter-relationships of the chronobiotic, melatonin, with leptin and adiponectin: implications for obesity. J Pineal Res. 2015;59(3):277-91 doi: https://doi.org/10.1111/jpi.12257

5. Rhee $\mathrm{YH}$, Ahn JC. Melatonin attenuated adipogenesis through reduction of the CCAAT/enhancer binding protein beta by regulating the glycogen synthase 3 beta in human mesenchymal stem cells. J Physiol Biochem. 2016;72(2):145-55. doi: https://doi.org/10.1007/s13105-015-0463-3

6. Jimenéz-Aranda A, Fernández-Vázquez G, Mohammad A-Serrano $M$, et al. Melatonin improves mitochondrial function in inguinal white adipose tissue of Zücker diabetic fatty rats. J Pineal Res. 2014;57(1):103-9. doi: https://doi.org/10.1111/jpi.12147

7. Fernández Vázquez G, Reiter RJ, Agil A. Melatonin increases brown adipose tissue mass and function in Zücker diabetic fatty rats: implications for obesity control. J Pineal Res. 2018;64(4):e12472. doi: https://doi.org/10.1111/jpi.12472

8. Xu P, Wang J, Hong F, et al. Melatonin prevents obesity through modulation of gut microbiota in mice. J Pineal Res. 2017;62(4). doi: https://doi.org/10.1111/jpi.12399.

9. Ireland KE, Maloyan A, Myatt L. Melatonin Improves Mitochondrial Respiration in Syncytiotrophoblasts From Placentas of Obese Women. Reprod Sci. 2018;25(1):120-130. doi: https://doi.org/10.1177/1933719117704908

10. Peschke E, Bähr I, Mühlbauer E. Melatonin and pancreatic islets: interrelationships between melatonin, insulin and glucagon. Int J Mol Sci. 2013;14(4):6981-7015. doi: https://doi.org/10.3390/ijms14046981

11. Qian J, Dalla Man C, Morris CJ, et al. Differential effects of the circadian system and circadian misalignment on insulin sensitivity and insulin secretion in humans. Diabetes Obes Metab. 2018;20(10):2481-2485. doi: https://doi.org/10.1111/dom.13391

12. Reiter RJ, Mayo JC, Tan DX, et al. Melatonin as an antioxidant: under promises but over delivers. J Pineal Res. 2016:61(3):253-78. doi: https://doi.org/10.1111/jpi.12360

13. Markwald RR, Melanson EL, Smith MR, et al. Impact of insufficient sleep on total daily energy expenditure, food intake, and weight gain. Proc Natl Acad Sci U S A. 2013;110(14):5695-700. doi: https://doi.org/10.1073/pnas.1216951110

14. Reiter RJ, Rosales-Corral S, Tan DX, et al. Melatonin as a mitochondria-targeted antioxidant: one of evolution's best ideas. Cell Mol Life Sci. 2017;74(21):3863-3881. doi: https://doi.org/10.1007/s00018-017-2609-7

15. Genario R, Morello E, Bueno AA, Santos HO. The usefulness of melatonin in the field of obstetrics and gynecology. Pharmacol Res. 2019;147:104337. doi: https://doi.org/10.1016/j.phrs.2019.104337

16. Fernandez B, Malde JL, Montero A, Acuña D. Relationship between adenohypophyseal and steroid hormones and variations in serum and urinary melatonin levels during the ovarian cycle, perimenopause and menopause in healthy women. J Steroid Biochem. 1990;35(2):257-62. doi: https://doi.org/10.1016/0022-4731(90)90282-w

17. Ma M, Chen XY, Li B, Li XT. Melatonin protects premature ovarian insufficiency induced by tripterygium glycosides: role of SIRT1. Am J Trans/ Res. 2017;9(4):1580-1602.

18. Tamura $\mathrm{H}$, Takasaki $\mathrm{A}$, Taketani $\mathrm{T}$, et al. Melatonin as a free radical scavenger in the ovarian follicle. Endocr J. 2013;60(1):1-13. doi: https://doi.org/10.1507/endocrj.ej12-0263

19. Tan DX, Manchester LC, Esteban-Zubero E, et al. Melatonin as a Potent and Inducible Endogenous Antioxidant: Synthesis and Metabolism. Molecules. 2015;20(10):18886-906. doi: https://doi.org/10.3390/molecules201018886

20. Nakamura $Y$, Tamura H, Takayama H, Kato H. Increased endogenous level of melatonin in preovulatory human follicles does not directly influence progesterone production. Fertil Steril. 2003;80(4):1012-6. doi: https://doi.org/10.1016/s0015-0282(03)01008-2

21. Teixeira AAC, Simöes MdJ, Teixeira VW, Soares Júnior JM. Evaluation of the implantation in pinealectomized and/or submitted to the constant illumination rats. Int. J. Morphol. 2004;22:189-194.

22. Song C, Peng W, Yin S, et al. Melatonin improves ageinduced fertility decline and attenuates ovarian mitochondrial oxidative stress in mice. Sci Rep. 2016;6:35165 doi: https://doi.org/10.1038/srep35165 
23. Tagliaferri V, Romualdi D, Scarinci E, et al. Melatonin Treatment May Be Able to Restore Menstrual Cyclicity in Women With PCOS: A Pilot Study. Reprod Sci. 2018;25(2):269-275. doi: https://doi.org/10.1177/1933719117711262

24. Genario R, Cipolla-Neto J, Bueno AA, Santos HO. Melatonin supplementation in the management of obesity and obesityassociated disorders: A review of physiological mechanisms and clinical applications. Pharmacol Res. 2021;163:105254 doi: https://doi.org/10.1016/j.phrs.2020.105254

25. Левин Я.И., Елигулашвили Т.С., Посохов С.И., и др. Фармакотерапия инсомний:роль Имована / В кн. Расстройства сна. Под ред. Ю.А. Александровского и А.М. Вейна. - СанктПетербург: Медицинское информационное агентство, 1995. - C. 56-61. [Levin Yal, Eligulashvili TS, Posokhov SI, et al. Farmakoterapiya insomnii: rol'Imovana. $V \mathrm{kn}$. Rasstroistva sna. Pod red. Yu.A. Aleksandrovskogo i A.M. Veina. Sankt-Peterburg: Meditsinskoe informatsionnoe agenstvo, 1995. S. 56-61 (in Russian)].

26. Summa KC, Turek FW. Chronobiology and obesity: Interactions between circadian rhythms and energy regulation. Adv Nutr. 2014;5(3):312S-9S. doi: https://doi.org/10.3945/an.113.005132

27. Corbalán-Tutau D, Madrid JA, Nicolás F, Garaulet M. Daily profile in two circadian markers «melatonin and cortisol» and associations with metabolic syndrome components. Physiol Behav. 2014;123:231-5. doi: https://doi.org/10.1016/j.physbeh.2012.06.005

28. Reiter RJ, Tan DX, Korkmaz A, Ma S. Obesity and metabolic syndrome: association with chronodisruption, sleep deprivation, and melatonin suppression. Ann Med. 2012;44(6):564-77. doi: https://doi.org/10.3109/07853890.2011.586365

29. Taheri S, Lin L, Austin D, et al. Short sleep duration is associated with reduced leptin, elevated ghrelin, and increased body mass index. PLoS Med. 2004;1(3):e62. doi: https://doi.org/10.1371/journal.pmed.0010062

30. Willis SK, Hatch EE, Wise LA. Sleep and female reproduction. Curr Opin Obstet Gynecol. 2019;31(4):222-227. doi: https://doi.org/10.1097/GCO.0000000000000554
31. Liu Y, Wheaton AG, Chapman DP, et al. Prevalence of Healthy Sleep Duration among Adults — United States, 2014. MMWR Morb Mortal Wkly Rep. 2016;65(6):137-41. doi: https://doi.org/10.15585/mmwr.mm6506a1

32. Wang Y, Gu F, Deng M, et al. Rotating shift work and menstrual characteristics in a cohort of Chinese nurses. BMC Womens Health. 2016;16:24. doi: https://doi.org/10.1186/s12905-016-0301-y

33. Kang W, Jang KH, Lim HM, et al. The menstrual cycle associated with insomnia in newly employed nurses performing shift work: a 12-month follow-up study. Int Arch Occup Environ Health. 2019;92(2):227-235. doi: https://doi.org/10.1007/s00420-018-1371-y

34. Goldstein CA, Lanham MS, Smith YR, O'Brien LM. Sleep in women undergoing in vitro fertilization: a pilot study. Sleep Med. 2017;32:105-113. doi: https://doi.org/10.1016/j.sleep.2016.12.007

35. Wang ID, Liu YL, Peng CK, et al. Non-Apnea Sleep Disorder Increases the Risk of Subsequent Female Infertility-A Nationwide Population-Based Cohort Study. Sleep. 2018;41(1). doi: https://doi.org/10.1093/sleep/zsx186

36. Willis SK, Hatch EE, Wesselink AK, et al. Female sleep patterns, shift work, and fecundability in a North American preconception cohort study. Fertil Steril. 2019;111(6):1201-1210.e1. doi: https://doi.org/10.1016/j.fertnstert.2019.01.037

37. Sturgeon SR, Doherty A, Reeves KW, et al. Urinary levels of melatonin and risk of postmenopausal breast cancer: women's health initiative observational cohort. Cancer Epidemiol Biomarkers Prev. 2014;23(4):629-37. doi: https://doi.org/10.1158/1055-9965.EPI-13-1028

38. Gómez-Acebo I, Dierssen-Sotos T, Papantoniou K, et al. Association between exposure to rotating night shift versus day shift using levels of 6-sulfatoxymelatonin and cortisol and other sex hormones in women. Chronobiol Int. 2015;32(1):128-35. doi: https://doi.org/10.3109/07420528.2014.958494

39. Luboshitzky R, Qupti G, Ishay A, et al. Increased 6-sulfatoxymelatonin excretion in women with polycystic ovary syndrome. Fertil Steril. 2001;76(3):506-10. doi: https://doi.org/10.1016/s0015-0282(01)01930-6

Рукопись получена: 01.12.2021. Одобрена к публикации: 27.02.2021. Опубликована online: 28.02.2022.

\section{ИНФОРМАЦИЯ ОБ АВТОРАХ [AUTHORS INFO]}

*Абсатарова Юлия Сергеевна, к.м.н. [Yulia S. Absatarova, MD, PhD]; адрес: 117036, г. Москва, ул. Дмитрия Ульянова, д. 11 [address: 11 Dm. Ulyanova street, 117036 Moscow, Russia]; ORCID: https://orcid.org /0000-0003-0696-5367, e-mail: korsil2008@yandex.ru

Андреева Елена Николаевна, д.м.н., профессор [Elena N. Andreeva, MD, PhD, professor], ORCID: http://orcid.org/0000-0001-8425-0020; SPIN-код: 1239-2937; e-mail: endogin@mail.ru Григорян Ольга Рафаэльевна, д.М.н., професcop [Olga R. Grigoryan, MD, PhD, professor]; ORCID: http://orcid.org/0000-0003-4979-7420; SPIN-код: 3060-8242; e-mail: iceberg1995@mail.ru

Шереметьева Екатерина Викторовна, к.M.H. [Ekaterina V. Sheremetyeva, MD, PhD]; ORCID: https://orcid.org/0000-0001-7177-0254; SPIN-код: 9413-5136; e-mail: s1981k@yandex.ru Михеев Роберт Константинович [Robert K. Mikheev, MD, resident]; ORCID: http://orcid.org/0000-0001-5826-3186; SPIN-код: 9767-8468; e-mail: iceberg1995@mail.ru

\section{ЦИТИРОВАТЬ:}

Андреева Е.Н., Григорян О.Р., Абсатарова Ю.С, Шереметьева Е.В., Михеев Р.К. Мелатониновый статус у пациенток с ожирением и дисфункцией яичников в репродуктивном возрасте // Проблемы эндокринологии. — 2022. — Т. 68. — №1. — C. 94-100. doi: https://doi.org/10.14341/probl12849

\section{TO CITE THIS ARTICLE:}

Andreeva EN, Grigoryan OR, Absatarova YS, Sheremetyeva EV, Mikheev RK. Melatonin status in obese patients with ovarian dysfunction at reproductive age. Problems of Endocrinology. 2022;68(1):94-100. doi: https://doi.org/10.14341/probl12849 